\title{
Cost Comparison of Flash Continuous Glucose Monitoring with Self-monitoring of Blood Glucose in Adults with Type 1 or Type 2 Diabetes Using Intensive Insulin-From a US Private Payer Perspective
}

\author{
Lizheng Shi' and Richard Hellmund² \\ 1. Tulane University, New Orleans, LA, USA; 2. Abbott Diabetes Care, Alameda, CA, USA
}

DOI: https://doi.org/10.17925/USE.2020.16.1.24

$\mathrm{O}$ bjective: Estimate the costs associated with a flash continuous glucose monitoring system as a replacement for self-monitoring of blood glucose (SMBG) for people using intensive insulin to manage diabetes, from a US private payer perspective. Methods: The base case used SMBG at 8 tests/day, the mid-point of the range recommended by the American Diabetes Association for intensive insulin users. Scenario analyses used SMBG at 3,6 and 10 tests/day. Estimated costs of glucose monitoring and severe hypoglycemic events were assessed. Results: For the base case, the annual cost of SMBG was $\$ 4,380$ for a patient with type 1 diabetes mellitus (T1DM) compared with $\$ 1,712$ for flash monitoring, a reduction of $\$ 2,667(61 \%)$. The cost of flash monitoring was also lower than SMBG at 6 and 10 tests/day. With SMBG at 3 tests/day the cost was comparable. Similar results were obtained for patients with T2DM using intensive insulin. The annual cost of severe hypoglycemia was approximately $50 \%$ less for flash monitoring than for SMBG for both patients with T1DM and those with T2DM. Conclusions: For US private payers, the flash monitoring system provides cost savings compared with SMBG for people with diabetes using intensive insulin. Cost savings with flash glucose monitoring may be realized through a reduction in severe hypoglycemia. Further study of budget impact is needed to include changes in behavior leading to improved adherence.

\section{Keywords}

Flash glucose monitoring, economics, type 1 diabetes, type 2 diabetes, US private payers, self-monitoring of blood glucose, continuous glucose monitoring, cost comparison

Disclosures: Lizheng Shi has no financial or non-financial relationships or activities to declare in relation to this article. Richard Hellmund is a full-time employee of Abbott Diabetes Care and is a stockholder.

Acknowledgments: Editorial assistance was provided by Patricia Forsyth of Touch Medical Media. This work was funded by Abbott Diabetes Care.

Review Process: Double-blind peer review.

Compliance with Ethics: This article reports a cost analysis and did not involve any direct studies with human or animal subjects performed by any of the authors.

Authorship: The named authors meet the criteria of the International Committee of Medical Journal Editors for authorship for this manuscript, take responsibility for the integrity of the work as a whole and have given final approval for the version to be published.

Access: This article is freely accessible at touchENDOCRINOLOGY.com (CTouch Medical Media 2020.

Received: December 17, 2019

Accepted: January 16, 2020

Published Online: April 2, 2020

Citation: US Endocrinology. 2020;16(1):24-30

Corresponding Author: Richard Hellmund,

Abbott Diabetes Care, 1420 Harbor Bay Parkway,

Alameda, CA 94502, USA. E: richard.hellmund@abbott.com

Support: The publication of this article was supported by Abbott Diabetes Care. The views and opinions expressed are those of the authors.

\section{Unmet needs for glucose monitoring in people with diabetes mellitus using intensive insulin}

Glucose monitoring is essential for people using intensive insulin to inform adjustments to the treatment and management of diabetes. Evidence suggests many people do not adhere to the recommended testing frequency with self-monitoring of blood glucose (SMBG). In the USA, it was estimated that people using intensive insulin test their glucose on average 2.6 times/day. ${ }^{1}$ This contrasts with the American Diabetes Association (ADA) Standards of Care, which recommend that people using intensive insulin regimens should test 6-10 times (or more) daily. Inadequate adherence to SMBG testing is associated with poor long-term outcomes, ${ }^{3}$ which, in turn, is likely to have a considerable economic impact. For example, there are significant costs associated with the medical treatment of hypoglycemic events. ${ }^{4}$ Published costs of hospital admissions in the USA for severe hypoglycemia range from $\$ 2,811-17,564$ per case, and tend to be higher for people with diabetic complications and comorbidities. 5.6

The main reasons for poor adherence to SMBG include a lack of time, not remembering to test, being self-conscious, pain and the perceived invasiveness of SMBG, and social stigma ${ }^{7 .}{ }^{78}$ Recently, continuous glucose monitoring (CGM) has overcome many of the barriers associated with SMBG and it can help people increase their adherence to the recommended frequency of glucose monitoring. ${ }^{9-11}$ For people with type 1 or type 2 diabetes mellitus (T1/T2DM) using intensive insulin, CGM has demonstrated improved glycemic control compared with SMBG in randomized controlled trials (RCTS). ${ }^{9-14}$ Despite significant clinical benefits, CGM is not widely used among the population of intensive insulin users, in part because of high acquisition costs, consequently leading to limited reimbursement. ${ }^{15}$ Out-of-pocket costs of CGM can also be high for people with insurance-based healthcare. ${ }^{16}$

\section{Flash continuous glucose monitoring}

The Freestyle Libre ${ }^{\mathrm{TM}}$ 14-day system (Abbott Diabetes Care, Alameda, CA, USA) is a new generation of CGM known as flash CGM. Flash monitoring has been available in the USA since 2017 for adults with diabetes, and it typically has lower acquisition costs than other current CGM systems."7 The system wirelessly transfers data from a sensor to a handheld reader (or smartphone). 
The sensor is applied to the back of the upper arm and lasts for up to 14 days. The system is factory-calibrated and does not require calibration using SMBG; the glucose data can also be used to make treatment decisions. ${ }^{\ddagger 18}$

The clinical benefit of flash monitoring for people using intensive insulin has been demonstrated in three RCTs. In two of these trials (IMPACT in people with T1DM, and REPLACE in people with T2DM), flash monitoring was associated with substantial decreases in daytime and nocturnal hypoglycemia compared with routine SMBG, without increasing glycated hemoglobin $(\mathrm{HbA} 1 \mathrm{C})$ or reducing the dose of insulin. 910 During the IMPACT and REPLACE trials, people using flash monitoring reduced their use of SMBG by $90 \%$, to 0.5 and 0.3 times/day, respectively, while scanning their sensors on average 15.1 and 8.3 times/day. All-cause health system resource use (hospital admissions, emergency room visits and ambulance use) was lower for people using flash monitoring compared with those using routine SMBG, especially in the REPLACE trial. The third RCT included people with T2DM, and demonstrated a reduction in $\mathrm{HDA} 1 \mathrm{C}$ and improved treatment satisfaction for flash monitoring compared with SMBG. ${ }^{14}$

In real-world studies, flash monitoring has demonstrated reductions from baseline in $\mathrm{HbA} 1 \mathrm{C}$ and hypoglycemia, ${ }^{19-29}$ and evidence from five countries shows that people who scan more frequently spend more time in range and have a lower mean glucose and estimated $\mathrm{HbA} 1 \mathrm{c} .^{30}$

Evidence supports the persistence of scanning outside the clinical trial setting. ${ }^{31}$ In an analysis based on 279,446 sensors, users performed a mean of 16.3 scans/day (median 14). In the REPLACE study, 125 of 139 patients completed the 6-month open-access phase (89.9\%). The average scan rate was 7.1 times/day (median 5.7) compared with 8.4 times/day (median 6.8) during the treatment phase, with $83.6 \%$ of possible glucose data collected compared with $88.7 \%$ during the treatment phase. ${ }^{31}$

Using UK National Health Service costs, flash monitoring has been shown to reduce health system costs when compared with routine SMBG. ${ }^{32,33}$ For people with T1DM using intensive insulin, the cost of flash monitoring was $19.4 \%$ less than SMBG at 10 tests/day, before allowing for potential reductions in costs associated with severe hypoglycemic events which would likely favor the flash monitoring system. ${ }^{32}$ For people with T2DM using intensive insulin, the cost of flash monitoring was $13.4 \%$ less than SMBG at 3 tests/day, once allowance was made for the costs of resource utilization reported in the REPLACE trial. ${ }^{33}$

\section{Objective}

The objective of this cost comparison was to estimate the costs associated with the flash monitoring system as a replacement for routine SMBG for people using intensive insulin to manage T1DM or T2DM. Acquisition costs of glucose monitoring and estimated costs of severe hypoglycemia have been included. The calculations were developed from the perspective of a us private payer.

\section{Materials and methods}

All calculations were conducted on a per patient per year (PPPY) basis.

־Based on the US Food and Drug Administration (FDA) label, SMBG tests remain required for treatment decisions when: 1) the Check Blood Glucose symbol is present; 2) symptoms do not match system readings; 3 ) the user suspects that readings may be inaccurate; 4) the user experiences symptoms that may indicate high or low blood glucose. ${ }^{18}$

\section{Acquisition cost of glucose monitoring for base case and different SMBG scenarios}

The acquisition cost of glucose monitoring was assessed at different frequencies of SMBG, based on the recommendation in the ADA Standards of Care that people using intensive insulin should monitor their glucose 6-10 times (or more) daily. ${ }^{2}$ The base case compared the annual cost per person for the flash monitoring system, with the cost of routine SMBG at 8 tests/day, the mid-point of the range recommended by the ADA. Scenarios 1 and 2 use the same approach as the base case, except they use the lower and upper limits of the range in the ADA Standards of Care, 6 and 10 SMBG tests/day, respectively. Scenario 3 is based on the same approach as the base case, but with routine SMBG used at 3 tests/day. Although much lower than the range recommended by the ADA, this is the frequency of SMBG observed in the REPLACE trial for people with T2DM using intensive insulin.

For the flash monitoring system, the calculations for the base case and different scenarios assumed a 14-day duration for each sensor, which is consistent with the sensor duration listed in the product labelling. ${ }^{18}$ This duration is supported by the median sensor duration of 13.92 days (interquartile range 13.61-13.95 days) observed in real-world use of the flash monitoring system, which was based on over 50,000 readers. ${ }^{17}$

Although the acquisition cost of the flash monitoring system is the same irrespective of whether people have T1DM or T2DM, separate cost calculations were created because there was a difference in the utilization of SMBG for people using flash monitoring in the IMPACT and REPLACE trials. For people with T1DM using intensive insulin, flash monitoring users were assumed to conduct a mean of 0.5 SMBG tests/day, as observed over the 6-month treatment period of the IMPACT trial. ${ }^{10}$ For people with T2DM using intensive insulin, flash monitoring users were assumed to conduct a mean of 0.3 SMBG tests/day, as observed over the 6-month treatment period of the REPLACE trial. ${ }^{9}$

The calculations used the list price for flash monitoring system sensors ( $\$ 54.43$ per 14 -day sensor) and readers ( $\$ 70.00$ per reader). Based on the factory warranty, each reader is assumed to last for 3 years and the cost was therefore assumed to be the same for each year ( $\$ 23.33 /$ year). Each lancet was assumed to cost $\$ 0.08$ and each test strip assumed to cost $\$ 1.42$, which was the mean of leading manufacturers' US list prices in January 2018.

\section{Potential costs of severe hypoglycemia}

For scenarios 1 and 3, evidence from the IMPACT and REPLACE trials was used to assess the potential costs of severe hypoglycemia. Scenario 1 was based on SMBG at 6 tests/day, which is close to the 5.6 SMBG tests/day observed in the IMPACT trial. Scenario 3 was based on SMBG at 3 tests/day, which is the same as the SMBG frequency observed in the REPLACE trial.

The average cost of treating a severe hypoglycemic event was estimated based on the proportions of events requiring an ambulance, use of an emergency room, or hospital admission, reported by Heller. ${ }^{4}$ These proportions were different for people with T1DM or T2DM. Consequently, the estimated cost for treating a severe hypoglycemic event for a person with T1DM was used for scenario 1, while the corresponding cost for treating a severe event for a person with T2DM was used for scenario 3. The costs for an ambulance, use of an emergency room, or a hospital 
admission are from a US Government Accountability Office report ${ }^{5}$ and a trial by Quilliam, ${ }^{\circ}$ updated to 2018 values based on the Consumer Price Index for medical care. ${ }^{34}$

In scenario 1, the rate of severe hypoglycemic events for routine SMBG users was assumed to be 3.20 events PPPY, based on an observational study of people with T1DM for over 15 years..$^{35}$ This evidence is from the UK; however, this source is considered suitable because it is based on an unselected population and hence is more relevant to the real-world setting of people with T1DM treated in general practice or by an endocrinologist. ${ }^{35}$ Although this source is from 2007, evidence from the EDIC study for 1995-2013 suggests that rates of severe hypoglycemia for people with T1DM using SMBG have been stable over time. ${ }^{36}$

Flash monitoring system users were assumed to experience a mean of 1.65 severe hypoglycemic events PPPY. This $48.5 \%$ reduction in the rate of events with the flash monitoring system was based on a proxy measure, the reduction in the rate of episodes with glucose levels below $45 \mathrm{mg} / \mathrm{dL}$ observed in the IMPACT trial. The use of changes in biochemical hypoglycemia as a proxy measure for changes in severe hypoglycemia is consistent with results reported using the data set from the Diabetes Control and Complications Trial, ${ }^{37}$ and is the same as the method used for a previous cost calculation. ${ }^{32}$

A similar approach was used for scenario 3, although in this case the rate of severe hypoglycemic events for routine SMBG users was assumed to be 1.05 events PPPY, based on a meta-analysis of trials of people with T2DM using insulin. ${ }^{38}$ Flash monitoring system users were assumed to experience a mean of 0.54 severe hypoglycemic events PPPY. This $48.8 \%$ reduction in the rate of events with flash monitoring was based on the reduction in the rate of episodes with glucose levels below $45 \mathrm{mg} / \mathrm{dL}$ observed in the REPLACE trial. ${ }^{9}$

\section{Budget impact based on hypothetical cohorts}

Hypothetical cohorts of 1,000 people using intensive insulin were used to assess the potential budget impact associated with the introduction of the flash monitoring system as a replacement for routine SMBG. Separate cohorts were considered for people with T1DM and for people with T2DM, and the base case utilization of SMBG, 8 tests/day, was assumed for people using SMBG. For both cohorts, everyone used routine SMBG in the baseline year; in the first year, $15 \%$ used the flash monitoring system and $85 \%$ used SMBG; in the second year, 25\% used flash monitoring and $75 \%$ used SMBG; and in the third year, 35\% used flash monitoring and $65 \%$ used SMBG. The total acquisition cost of glucose monitoring was estimated for each year.

\section{Results}

\section{Acquisition cost of glucose monitoring}

The acquisition cost of routine SMBG for the base case (8 tests/day), was estimated to be \$4,380 PPPY compared with \$1,712 PPPY for people with T1DM using the flash monitoring system, a reduction of $\$ 2,667$ (61\%). The cost for flash monitoring includes \$274 PPPY for SMBG, based on 0.5 tests/day observed in the IMPACT trial. For people with T2DM, the acquisition cost for the flash monitoring system was \$1,603 PPPY, a reduction of $\$ 2,777$ (63\%) compared with routine SMBG. The cost for flash monitoring includes \$164 PPPY for SMBG, based on 0.3 tests/day observed in the REPLACE trial.
Table 1: Acquisition cost of glucose monitoring for the flash monitoring system compared with self-monitoring of blood glucose at 3, 6, 8, and 10 tests/day

\begin{tabular}{|c|c|c|c|}
\hline & $\begin{array}{l}\text { T1DM using } \\
\text { intensive insulin }\end{array}$ & Frequency & $\begin{array}{l}\text { T2DM using } \\
\text { intensive insulin }\end{array}$ \\
\hline \multicolumn{4}{|c|}{ Flash monitoring system, \$ } \\
\hline Cost per reader & 70.00 & $\begin{array}{l}1 \text { reader every } \\
3 \text { years }\end{array}$ & 70.00 \\
\hline Cost per 14-day sensor & 54.43 & $\begin{array}{l}26 \text { sensors } \\
\text { per year }\end{array}$ & 54.43 \\
\hline $\begin{array}{l}\text { Cost of reader and } \\
\text { sensor, PPPY }\end{array}$ & $1,438.51$ & & $1,438.51$ \\
\hline \multicolumn{4}{|l|}{ SMBG, \$ } \\
\hline Cost per lancet & 0.08 & & 0.08 \\
\hline Cost per test strip & 1.42 & & 1.42 \\
\hline $\begin{array}{l}\text { Cost of lancet }+ \text { test } \\
\text { strip }\end{array}$ & 1.50 & & 1.50 \\
\hline $\begin{array}{l}\text { For flash monitoring } \\
\text { system users, PPPY }\end{array}$ & 273.75 & $\begin{array}{l}0.5 \mathrm{SMBG} \\
\text { tests/day (T1DM) } \\
0.3 \mathrm{SMBG} \\
\text { tests/day (T2DM) }\end{array}$ & 164.25 \\
\hline \multirow[t]{4}{*}{$\begin{array}{l}\text { For routine SMBG users, } \\
\text { PPPY }\end{array}$} & $4,380.00$ & $\begin{array}{l}8 \text { SMBG tests/day } \\
\text { (base case) }\end{array}$ & $4,380.00$ \\
\hline & $1,642.50$ & 3 SMBG tests/day & $1,642.50$ \\
\hline & $3,285.00$ & 6 SMBG tests/day & $3,285.00$ \\
\hline & $5,475.00$ & $\begin{array}{l}10 \text { SMBG } \\
\text { tests/day }\end{array}$ & $5,475.00$ \\
\hline $\begin{array}{l}\text { Cost of flash monitoring } \\
\text { system, PPPY }\end{array}$ & $1,712.26$ & & $1,602.76$ \\
\hline
\end{tabular}

PPPY = per patient per year; $S M B G=$ self-monitoring of blood g/ucose; T1DM = type 1 diabetes mellitus; T2DM = type 2 diabetes mellitus.

Scenarios 1 and 2 also demonstrated substantial cost savings for flash monitoring compared with SMBG at 6 and 10 tests/day for people with T1DM or T2DM, ranging from \$1,573-3,872 PPPY. For scenario 3, there was a small cost reduction for flash monitoring compared with SMBG for people with T2DM. There was a small cost increase for flash monitoring in scenario 3 for people with T1DM, although the additional cost in this scenario is much less than the cost savings shown in the base case or other scenarios.

Based on the results for the base case and scenarios, it is concluded that for people with diabetes who are using intensive insulin, the acquisition cost of glucose monitoring using the flash monitoring system is substantially less than that for routine SMBG testing from 3-10 or more times/day (Table 1, Figures $1 A$ and $1 B$ ).

\section{Estimated cost of severe hypoglycemia}

The estimated average cost of treating a severe hypoglycemic event was \$1,404 for people with T1DM using intensive insulin and \$1,671 for people with T2DM using intensive insulin (Table 2). For people with T1DM using intensive insulin, Table 3 and Figure 2 shows the cost of severe hypoglycemia for the flash monitoring system was estimated at $\$ 2,317$ PPPY, compared with $\$ 4,494$ PPPY for routine SMBG. This is a reduction of $\$ 2,177$ PPPY in favor of the flash monitoring system, together with a reduction in the incidence of severe hypoglycemia of almost $50 \%$. 
Figure 1: Reduction in acquisition costs of flash monitoring compared with SMBG, PPPY in patients with type 1 diabetes (A) and type 2 diabetes (B)

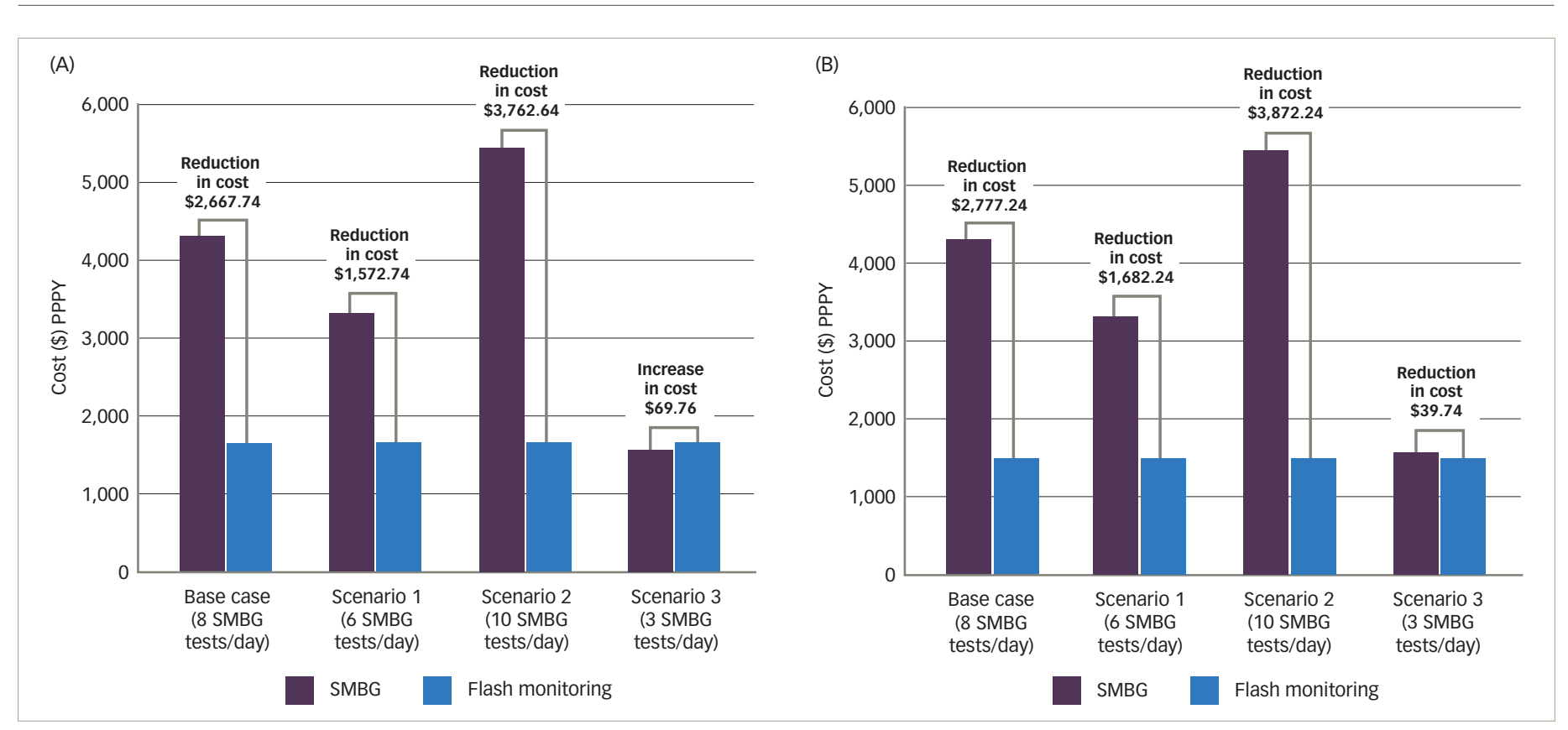

$P P P Y=$ per patient per year; $S M B G=$ self-monitoring of blood g/ucose .

Table 2: Average cost of a severe hypoglycemic event for people with type 1 or type 2 diabetes using intensive insulin

\begin{tabular}{|l|l|l|l|}
\hline & $\begin{array}{l}\text { \% of severe events requiring } \\
\text { this resource }\end{array}$ & Cost per event (\$) & $\begin{array}{l}\text { Average cost per severe } \\
\text { hypoglycemic event (\$) }\end{array}$ \\
\hline T1DM using intensive insulin & 31.0 & 542.69 & 168.23 \\
\hline Ambulance & 9.5 & $1,697.44$ & 161.26 \\
\hline Emergency room & 5.0 & $21,498.64$ & $1,074.93$ \\
\hline Hospital admission & & & $\mathbf{1 , 4 0 4 . 4 2}$ \\
\hline Average cost of a severe hypoglycemic event & 25.3 & 542.69 & 137.30 \\
\hline T2DM using intensive insulin & 23.2 & $1,697.44$ & 393.81 \\
\hline Ambulance & 5.3 & $21,498.64$ & $1,139.43$ \\
\hline Emergency room & & & $\mathbf{1 , 6 7 0 . 5 4}$ \\
\hline Hospital admission & & & \\
\hline Average cost of a severe hypoglycemic event & & \\
\hline
\end{tabular}

T1DM = type 1 diabetes mellitus; T2DM = type 2 diabetes mellitus.

For people with T2DM using intensive insulin, Table 3 and Figure 2 shows the cost of severe hypoglycemia for the flash monitoring system was estimated at \$902 PPPY, compared with \$1,754 PPPY for routine SMBG. This is a reduction of $\$ 852$ PPPY in favor of the flash monitoring system together with a reduction in the incidence of severe hypoglycemia of almost $50 \%$.

\section{Combined cost of glucose monitoring and severe hypoglycemia}

For scenario 1, the flash monitoring system was compared with SMBG at 6 tests/day, which is close to the 5.6 tests/day observed in the IMPACT trial. For people with T1DM using intensive insulin, the cost savings associated with use of flash monitoring compared with routine SMBG (at 6 tests/day) was $\$ 3,750$ PPPY. These savings were based on a reduction of \$1,573 PPPY in the cost of glucose monitoring and \$2,177 PPPY in the cost of medical treatment of severe hypoglycemic events. For scenario 3 , the flash monitoring system was compared with SMBG at 3 tests/day, which is the same as the 3.0 tests/day observed in the REPLACE trial. For people with T2DM using intensive insulin, the cost savings associated with use of flash monitoring compared with routine SMBG at 3 tests/day was $\$ 892$ PPPY. These savings were based on a reduction of \$40 PPPY in the cost of glucose monitoring and \$852 PPPY in the cost of medical treatment of severe hypoglycemic events.

For both these scenarios, the savings associated with use of flash monitoring were even greater from reductions in severe hypoglycemia than from glucose monitoring. This confirms that on a per-patient basis, the overall cost of using the flash monitoring system will be less than for routine SMBG for people with T1DM or T2DM who are using intensive insulin. 
Table 3: Estimated cost of severe hypoglycemic events for people with type 1 and type 2 diabetes using intensive insulin

\begin{tabular}{|l|l|l|}
\hline \multicolumn{2}{|l|}{$\begin{array}{l}\text { T1DM using } \\
\text { intensive insulin }\end{array}$} & $\begin{array}{l}\text { T2DM using } \\
\text { intensive insulin }\end{array}$ \\
\hline SMBG & 3.20 & 1.05 \\
\hline $\begin{array}{l}\text { Estimated rate of severe hypoglycemic } \\
\text { events, PPPY }\end{array}$ & $1,404.42$ & $1,670.54$ \\
\hline $\begin{array}{l}\text { Average cost of a severe hypoglycemic } \\
\text { event (\$) }\end{array}$ & $4,494.14$ & $1,754.07$ \\
\hline $\begin{array}{l}\text { Estimated cost of severe hypoglycemic } \\
\text { events, PPPY (\$) }\end{array}$ & 48.5 & 48.8 \\
\hline \begin{tabular}{l} 
Flash monitoring \\
\hline $\begin{array}{l}\text { Proxy for reduction in rate of severe } \\
\text { hypoglycemic events versus SMBG,b (\%) }\end{array}$
\end{tabular} & 1.65 & 0.54 \\
\hline $\begin{array}{l}\text { Estimated rate of severe hypoglycemic } \\
\text { events, PPPY }\end{array}$ & $1,404.42$ & $1,670.54$ \\
\hline $\begin{array}{l}\text { Average cost of a severe hypoglycemic } \\
\text { event (\$) }\end{array}$ & $2,317.29$ & 902.09 \\
\hline $\begin{array}{l}\text { Estimated cost of severe hypoglycemic } \\
\text { events, PPPY (\$) }\end{array}$ & $2,176.85$ & 851.98 \\
\hline $\begin{array}{l}\text { Reduction in cost for flash monitoring } \\
\text { system compared with SMBG, PPPY, \$ }\end{array}$ & & \\
\hline
\end{tabular}

${ }^{a}$ Assumption: reduction in rate of episodes $<45 \mathrm{mg} / \mathrm{dL}$, observed in the IMPACT study with SMBG at 5.6 tests/day. ${ }^{10}$

${ }^{b}$ Assumption: reduction in rate of episodes $<45 \mathrm{mg} / \mathrm{dL}$, observed in the REPLACE study with SMBG at 3.0 tests/day. ${ }^{9}$

$P P P Y=$ per patient per year; $S M B G=$ self-monitoring of blood g/ucose

Figure 2: Reduction in estimated costs of severe hypoglycemic events for patients with type 1 and type 2 diabetes

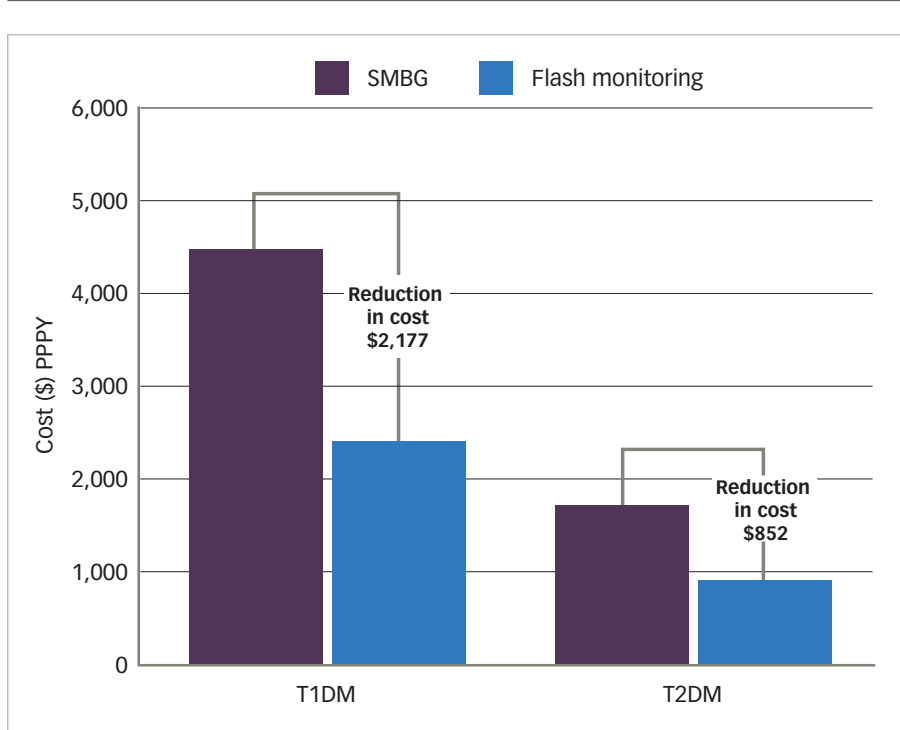

$P P P Y=$ per patient per year; $S M B G=$ self-monitoring of blood glucose; T1DM = type 1 diabetes mellitus; T2DM = type 2 diabetes mellitus.

\section{Budget impact based on hypothetical cohorts}

For a cohort of 1,000 people with T1DM using intensive insulin, the total cost of glucose monitoring decreased from $\$ 4.38$ million in the baseline year to $\$ 3.45$ million in year 3 (Table 4 ). This is a reduction of $21 \%$ in the cost of glucose monitoring over the 3-year period. Similarly, for a cohort of 1,000 people with T2DM using intensive insulin, the total cost of glucose monitoring decreased from $\$ 4.38$ million in the baseline year, to $\$ 3.41$ million in year 3 (Table 4). This is a reduction of $22 \%$ in the cost of glucose monitoring over the 3-year period. These savings do not include additional savings from potential reductions in the rate of severe hypoglycemic events associated with the flash monitoring system.

\section{Discussion}

From a US private payer perspective, for people with diabetes and who are using intensive insulin, these cost calculations demonstrate the flash CGM system provides a substantial cost-saving compared with SMBG testing from 3-10 or more times/day. In addition, the scanning rates observed in the IMPACT and REPLACE trials show that flash monitoring is associated with improved adherence to the recommended testing frequency from the ADA Standards of Care when compared with routine SMBG.9.10 Real-world evidence confirms that the scanning frequency observed in the IMPACT and REPLACE trials is maintained when flash monitoring is used by people in the regular daily management of their diabetes. ${ }^{32}$ Therefore, compared with SMBG, the flash monitoring system is expected to reduce the number of severe hypoglycemic events, reduce health system costs, and achieve greater adherence to recommended glucose monitoring frequencies for people with diabetes who are using intensive insulin.

The improvement in adherence to the recommended glucose monitoring frequency with the flash monitoring system may be higher than suggested by studies of SMBG. ${ }^{1}$ This is because those studies are typically based on prescription record data, which tends to over-estimate actual utilization of SMBG.

The possibility that use of the flash monitoring system could lead to reductions in health system costs is also supported by secondary evidence from the REPLACE trial, which showed a large reduction in utilization of health system resources (emergency room visits, ambulance callouts and hospital admissions) compared with the SMBG group, on an all-cause basis. ${ }^{33}$

The benefits of flash monitoring are supported by the observation that each scan provides more information than a single glucose reading from a SMBG test. For example, the flash monitoring system can provide a summary ambulatory glucose profile and a complete 24-hour glucose record. With flash monitoring, users are encouraged to test their glucose frequently because the incremental cost of each scan is zero, unlike SMBG where supplies are typically limited for each patient and the health system will pay more for an increased testing frequency. ADA Standards of Care and evidence from real-world studies support frequent glucose testing to achieve effective management of both $\mathrm{HbA1c}$ levels and to reduce the risk of hypoglycemia associated with intensive insulin use; flash monitoring appears to be more consistent with this goal than SMBG. ${ }^{2,3}$

A recent international consensus statement endorsed by the ADA, American Association of Clinical Endocrinologists, American Association of Diabetes Educators, European Association for the Study of Diabetes, and the International Society for Pediatric and Adolescent Diabetes, ${ }^{39}$ recognizes the importance of time in glucose ranges (TIR) as "appropriate and useful as clinical targets and outcome measurements". The flash monitoring system provides TIR in the ambulatory glucose profile report; however, SMBG does not provide this valuable measure and therefore is not consistent with the international consensus statement in this regard. 
Table 4: Impact on a hypothetical budget of the introduction of the flash monitoring system for patients with type 1 or type 2 diabetes using intensive insulin

\begin{tabular}{|c|c|c|c|c|}
\hline & $\begin{array}{l}\text { Year } 0 \\
\text { (Baseline) }\end{array}$ & Year 1 & Year 2 & Year 3 \\
\hline \multicolumn{5}{|l|}{ T1DM using intensive insulin } \\
\hline \% using SMBG at 8 tests/day; \% using flash monitoring & $100 ; 0$ & $85 ; 15$ & $75 ; 25$ & $65 ; 35$ \\
\hline Cost of SMBG (8 tests/day, \$ millions) & $\$ 4.38$ & $\$ 3.72$ & $\$ 3.29$ & $\$ 2.85$ \\
\hline Cost of flash monitoring (\$ millions) & $\$ 0.00$ & $\$ 0.26$ & $\$ 0.43$ & $\$ 0.60$ \\
\hline Total cost (\$ millions per 1,000 patient cohort) & $\$ 4.38$ & $\$ 3.98$ & $\$ 3.72$ & $\$ 3.45$ \\
\hline \multicolumn{5}{|l|}{ T2DM using intensive insulin } \\
\hline \% using SMBG at 8 tests/day; \% using flash monitoring & $100 ; 0$ & $85 ; 15$ & $75 ; 25$ & $65 ; 35$ \\
\hline Cost of SMBG (8 tests/day, \$ millions) & $\$ 4.38$ & $\$ 3.72$ & $\$ 3.29$ & $\$ 2.85$ \\
\hline Cost of flash monitoring ( $\$$ millions) & $\$ 0.00$ & $\$ 0.24$ & $\$ 0.40$ & $\$ 0.56$ \\
\hline Total cost ( $\$$ millions per 1,000 patient cohort) & $\$ 4.38$ & $\$ 3.96$ & $\$ 3.69$ & $\$ 3.41$ \\
\hline
\end{tabular}

$S M B G$ = self-monitoring of blood g/ucose; T1DM = type 1 diabetes mellitus; $T 2 D M=$ type 2 diabetes mellitus.

Table 5: Number and rate of hospitalizations among adults aged $\geq 18$ years with diabetes, USA, $2016^{41}$

\begin{tabular}{|l|l|l|}
\hline Cause of hospitalization & $\begin{array}{l}\text { Number, in } \\
\text { thousands }\end{array}$ & $\begin{array}{l}\text { Crude rate per } \\
1,000 \text { persons with } \\
\text { diabetes (95\% Cl) }\end{array}$ \\
\hline Diabetes as any listed diagnosis & 7,833 & $339.0(317.6-360.4)$ \\
\hline Major cardiovascular disease & 1,740 & $75.3(70.4-80.2)$ \\
\hline Ischemic heart disease & 438 & $18.9(17.6-20.2)$ \\
\hline Stroke & 313 & $13.6(12.7-14.5)$ \\
\hline Lower-extremity amputation & 130 & $5.6(5.3-6.0)$ \\
\hline Diabetic ketoacidosis & 188 & $8.1(7.6-8.7)$ \\
\hline
\end{tabular}

$\mathrm{Cl}=$ confidence interval.

Data source: United States Diabetes Surveillance System.

Poor glucose control is associated with an increased risk of microvascular complications, while severe hypoglycemiaisassociated with cardiovascular disease..$^{40}$ Treating the complications of diabetes imposes a substantial burden on health systems in the USA. Table 5 presents evidence from the Centers for Disease Control and Prevention that in 2016 there were an estimated 7,833,000 hospitalizations for adults with diabetes, including $1,740,000$ for major cardiovascular disease. ${ }^{41}$ Potentially, the reduction in hypoglycemia provided by the flash monitoring system may lead in the longer term to reductions in the incidence of cardiovascular events or other complications, reducing associated healthcare costs.

Possible limitations of the cost calculations in the present study include the exclusion of the cost of insulin and the focus on direct costs to the health system. Although the cost of insulin is an important consideration, it was not included in the calculations here because the IMPACT and REPLACE trials showed that insulin use was similar in the flash monitoring and SMBG arms., ${ }^{9,10}$ These cost calculations are restricted to direct treatment costs and do not include the wider costs of diabetes managed with intensive insulin. These include the possible costs, clinical consequences, and productivity losses associated with non-severe hypoglycemic events.
Concerning resources related to severe hypoglycemia, only clearly stated resource use was included. ${ }^{4}$ The authors acknowledge there was potential for under-reporting of ambulance use; also additional blood glucose measurements, costs of glucagon or intravenous dextrose, or any subsequent medical consultations that were necessary following severe hypoglycemia were not included. Given the substantial reduction in hypoglycemia observed in the IMPACT and REPLACE trials, it is possible that flash monitoring may reduce these indirect costs in the population of intensive insulin users. These cost calculations also do not take account of resource use associated with hyperglycemia, including diabetic ketoacidosis (DKA); recent real-world evidence shows flash monitoring reduces the rate of DKA compared to the period before flash monitoring was initiated, ${ }^{42,43}$ which may provide additional cost savings.

Overall, the estimated resource use in these cost calculations may be an under-estimate of the underlying total resource use for flash monitoring and SMBG, and the cost savings for flash monitoring compared with SMBG may be greater than stated here. Although these cost calculations have focused on the private payer, a previous assessment has been reported of the cost of flash monitoring versus routine SMBG in intensive insulin users with T2DM covered by Medicare and Medicaid. ${ }^{44}$ This assessment did not make allowance for the benefit of flash monitoring in reducing health system costs compared with SMBG by avoiding severe hypoglycemic events. Allowing for this factor, the flash monitoring system could potentially be cost neutral in Medicare and Medicaid populations, while increasing adherence to ADA monitoring guidelines and reducing the number of hospitalizations required for severe hypoglycemia.

The annual cost per patient of the flash monitoring system in these calculations is $\$ 1,712$, which compares favorably with the annual cost reported for other CGM systems. For example, in 2016, a US model used an annual cost for CGM of $\$ 4,500-4,900,{ }^{45}$ while a UK model included a cost of $£ 3,740^{46}(\$ 4,600)$ and a Canadian model used $C \$ 6,588$ $(\$ 4,940){ }^{47}$ This cost advantage is supported by ADA Standards of Care, which state that flash monitoring "may be a more affordable alternative to real-time CGM systems for patients on intensive insulin therapy." ${ }^{2}$ 
In addition, the flash monitoring system is approved by the US Food and Drug Adminstration as nonadjunctive, meaning it is designed to replace SMBG. ${ }^{\ddagger}$ Some CGM systems, in contrast, still require the additional expense of SMBG to enable users to make insulin-dosing decisions.

\#Based on the FDA label, SMBG tests remain required for treatment decisions when: 1) the Check Blood Glucose symbol is present; 2) symptoms do not match system readings; 3 ) the user suspects that readings may be inaccurate; 4) the user experiences symptoms that may indicate high or low blood glucose.

\section{Conclusion}

These calculations demonstrate that, from a US private payer perspective, the flash CGM system provides cost savings compared with routine SMBG for people with diabetes who are using intensive insulin. Scenarios show that cost savings with flash monitoring are achievable compared with SMBG from 3-10 or more tests/day. Cost savings with flash glucose monitoring may be realized through a reduction in severe hypoglycemia. Further study of budget impact is needed to include changes in behavior leading to improved adherence.
1. Yeaw J, Lee WC, Aagren M, Christensen T. Cost of self-monitoring of blood glucose in the United States among patients on an insulin regimen for diabetes. J Manag Care Pharm. 2012; 18:21-32.

2. American Diabetes Association. 7. Diabetes Technology: Standards of Medical Care in Diabetes-2019. Diabetes Care 2019:42(Suppl. 1):S71-80

3. Miller KM, Beck R, Bergenstal R, et al. Evidence of a strong association between frequency of self-monitoring of blood glucose and hemoglobin A1c levels in T1D exchange clinic registry participants. Diabetes Care. 2013;36:2009-14.

4. Heller S, Frier B, Hersløv M, et al. Severe hypoglycaemia in adults with insulin-treated diabetes: impact on healthcare resources. Diabet Med 2016:33:471-7.

5. United States Government Accountability Office (GAO). Report to Congressional Committees: Ambulance providers - Costs and Medicare margins varied widely; transports of beneficiaries hav increased. 2012. Available at: www.gao.gov/assets/650/649018. pdf (accessed March 2, 2020)

6. Quilliam B, Simeone J, Ozbay AB, et al. The incidence and costs of hypoglycemia in type 2 diabetes. Am J Manag Care. 2011:17:673-80.

7. Ong WM, Chua SS, Ng CJ. Barriers and facilitators to self-monitoring of blood glucose in people with type 2 diabetes using insulin: a qualitative study. Patient Prefer Adherence. 2014;8:237-46

8. Hellmund R. Self-assessment of glucose levels in the real world is less frequent than is proposed in major guidelines. Diabetes. 2015;64(Suppl. 1):A235-382. Abstr 973-P.

9. Haak T, Hanaire H, Ajian R, et al. Flash glucose-sensing technology as a replacement for blood glucose monitoring for the management of insulin-treated type 2 diabetes: a multicenter, open-label randomized controlled trial. Diabetes Ther. 2017;8:55-73.

10. Bolinder J, Antuna R, Geelhoed-Duijvestijn P, et al. Novel glucose-sensing technology and hypoglycaemia in type diabetes: a multicentre, non-masked, randomised controlled tria (IMPACT). Lancet. 2016;388:2254-63.

11. Beck R, Riddlesworth $T$, Ruedy $K$, et al. Effect of continuous glucose monitoring on glycemic control in adults with type 1 diabetes using insulin injections: the DIAMOND randomized clinical trial. JAMA. 2017;317:371-8.

12. Lind $\mathrm{M}$, Polonsky W, Hirsch IB, et al. Continuous glucose monitoring vs conventional therapy for glycemic control in adults with type 1 diabetes treated with multiple daily insulin injections the GOLD randomized clinical trial. JAMA. 2017:317:379-87.

13. Beck RW, Riddlesworth $\mathrm{T}$, Ruedy $\mathrm{K}$, et al. Continuous glucose monitoring versus usual care in patients with type 2 diabetes receiving multiple daily insulin injections: a randomized trial. An Intern Med. 2017;167:365-74.

14. Yaron M, Roitman E, Aharon-Hananel G, et al. Effect of flash glucose monitoring technology on glycemic control and treatment satisfaction in patients with type 2 diabetes. Diabetes Care. 2019:42:1178-84.

15. YU S, Varughese B, Li Z, Kushner PR. Healthcare resource waste associated with patient nonadherence and early discontinuation of traditional continuous glucose monitoring in real-world settings: a multicountry analysis. Diabetes Technol Ther. 2018;20:420-7

16. Hellmund R. A review of out-of-pocket costs for people using continuous glucose monitoring in the United States. Presented at:
American Association of Diabetes Educators Annual Conference, Houston, TX, USA, August 9-12, 2019. Poster IP02.

17. Abbott Diabetes Care, data on file.

18. Freestyle Libre 14 Day Flash Glucose Monitoring System User's Manual. 2018. Available at: www.accessdata.fda.gov/cdrh docs/ pdf16/P160030S017C.pdf (accessed March 2, 2020).

19. Evans $M$, Welsh Z, Ells S, Seibold A. The impact of flash glucose monitoring on glycaemic control as measured by HDA1c: a meta-analysis of clinical trials and real-world observational studies. Diabetes Ther. 2019;11:83-95.

20. Löndahl M, Berntrop K, Filipsson K, et al. Flash glucose monitoring improves metabolic control and treatment satisfaction in people with type 1 diabetes. Presented at: 52nd Annual Meeting of the European Association for the Study of Diabetes, Munich, Germany, September 12-6, 2016. Abstr. 871

21. Löndahl $M$, Filipsson $K$, Lindholm E, Katzman P. Effect of flash glucose monitoring on metabolic control and self-esteemed treatment satisfaction in people with type 1 diabetes. Diabetes Technol Ther. 2017;19:A-81.

22. McKnight JA, Gibb FW. Flash glucose monitoring is associated with improved glycaemic control but use is largely limited to more affluent people in a UK diabetes centre. Diabet Med. 2017:34:732.

23. Löndahl M, Berntrop K, Filipsson $\mathrm{K}$, et al. Flash glucose monitoring improves metabolic control and treatment satisfaction in people with type 1 diabetes [Abstract]. Diabetologia. 2016;59:871.

24. Ish-Shalom M, Wainstein J, Raz I, Mosenzon O. Improvement in glucose control in difficult-to-control patients with diabetes using a novel flash glucose monitoring device. J Diabetes Sci Technol. 2016:10:1412-3.

25. Holcombe A, Karunakaran V Streeting J, et al. Trial of Freestyle Libre in a local service: impact on diabetes outcomes [Poster P418]. Diabetic Medicine. 2017;34(Suppl. 1):159-63.

26. Dover AR, Stimson RH, Zammitt NN, Gibb FW. Flash glucose monitoring improves outcomes in a type 1 diabetes clinic. J Diabetes Sci Technol. 2017;11:442-3.

27. Paris I, Henry C, Gérard A, et al. Le Freestyle Libre en Pratique Clinique: Une Expérience Belge [Poster]. Presented at: Congrès Annuel de la Société Francophone du Diabète, Lille, France, March 28-31, 2017

28. Deshmukh $H$, Wilmot E, Bishop D, et al. The Association of British Clinical Diabetologists UK-wide audit of Freestyle Libre use in diabetes-effect on glycaemic control. Diabetes. 2019;68(Suppl. 1):299.

29. Wilmot E, Deshmukh $H$, Bishop D, et al. The Association of British Clinical Diabetologists' audit of Freestyle Libre in diabetes in United Kingdom-effect on hypoglycaemia awareness. Diabetes. 2019:68(Suppl. 1):959.

30. Dunn T, Xu Y, Hayter G, Ajian R. Real-world flash glucose monitoring patterns and associations between self-monitoring frequency and glycaemic measures: a European analysis of over 60 million glucose tests. Diabetes Res Clin Pract. 2018;137:37-46.

31. Haak $\mathrm{T}$, Hanaire $\mathrm{H}$, Ajjan $\mathrm{R}$, et al. Use of flash glucose-sensing technology for 12 months as a replacement for blood glucose monitoring in insulin-treated type 2 diabetes. Diabetes Ther. 2017;8:573-86.

32. Hellmund R, Weitgasser R, Blissett D. Cost calculation for a flash glucose monitoring system for UK adults with type 1 diabetes mellitus receiving intensive insulin treatment. Diabetes Res Clin Pract. 2018;138:193-200.

33. Hellmund R, Weitgasser R, Blissett D. Cost calculation for a flash glucose monitoring system for adults with type 2 diabetes mellitus using intensive insulin - a UK perspective. Eur Endocrinol. 2018;14:86-92.

34. Health Resources and Services Administration. Consumer Price Index (CPI) for Medical Care. 2019. Available at: www.hrsa.gov/ get-health-care/affordable/hill-burton/cpi.html (accessed March 3, 2020)

35. UK Hypoglycaemia Study Group. Risk of hypoglycaemia in types 1 and 2 diabetes: effects of treatment modalities and their duration Diabetologia. 2007; 50:1140-7.

36. Gubitosi-Klug RA, Braffett BH, White NH, et al. Risk of severe hypoglycemia in type 1 diabetes over 30 years of follow-up in the DCCT/EDIC study. Diabetes Care. 2017;40:1010-6.

37. Beck RW, Bergenstal RM, Riddlesworth TD, et al. The association of biochemical hypoglycemia with the subsequent risk of a severe hypoglycemic event: analysis of the DCCT data set. Diabetes Technol Ther. 2019;21:1-5.

38. Edridge $\mathrm{CL}$, Dunkley AJ, Bodicoat $\mathrm{DH}$, et al. Prevalence and incidence of hypoglycaemia in 532,542 people with type 2 diabetes on oral therapies and insulin: a systematic review. and meta-analysis of population based studies. PLOS One. 2015;10:e0126427.

39. Battelino T, Danne T, Bergensta RM, et al. Clinical targets for continuous glucose monitoring data interpretation: recommendations from the international consensus on time in range. Diabetes Care. 2019;42:1593-603

40. Goto A, Arah OA, Goto M, et al. Severe hypoglycaemia and cardiovascular disease: systematic review and meta-analysis with bias analysis. BMJ. 2013:347:f4533.

41. Centers for Disease Control and Prevention. National Diabetes Statistics Report. Estimates of diabetes and its burden in the United States. 2020. Available at: www.cdc.gov/diabetes/pdfs/ data/statistics/national-diabetes-statistics-report.pdf (accessed March 3, 2020)

42. Tyndall V, Stimson RH, Zammitt NN, et al. Marked improvement in $\mathrm{HbA1C}$ following commencement of flash glucose monitoring in people with type 1 diabetes. Diabetologia. 2019;62:1349-56.

43. Charleer S, De Block C, Van Huffel L, et al. Quality of life and glucose control after 1 year of nationwide reimbursement of intermittently scanned continuous glucose monitoring in adults living with type 1 diabetes (FUTURE): a prospective observational real-world cohort study. Diabetes Care. 2020;43:389-97.

44. Stueve M, Zoellner Y. Budget impact analysis of self-monitoring of blood glucose vs. flash-continuous glucose monitoring in intensive insulin users with diabetes type 2 covered by Medicare and Medicaid. Diabetes. 2018;67(Suppl. 1). DOI: 10.2337/db18142-LB.

45. Bronstone A, Graham C. The potential cost implications of averting severe hypoglycemic events requiring hospitalization in high-risk adults with type 1 diabetes using real-time continuous glucose monitoring. I Diabetes Sci Technol. 2016;10:905-13.

46. Chaugule S, Oliver N, Klinkenbill B, Graham C. An economic evaluation of continuous glucose monitoring for people with type 1 diabetes and impaired awareness of hypoglycaemia within North West London Clinical Commissioning Groups in England. Eur Endocrinol. 2017;13:81-5.

47. Chaugule S, Graham C. Cost effectiveness of G5 Mobile continuous glucose monitoring (CGM) device compared with self-monitoring of blood glucose (SMBG) alone for people with Type 1 diabetes from the Canadian societal perspective. J Med ECon. 2017;20:1128-35. 